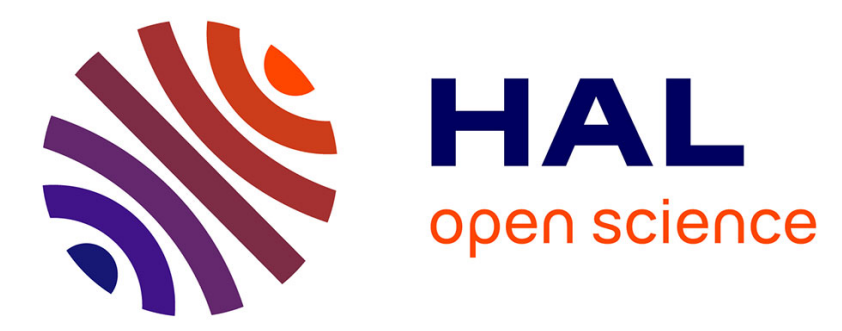

\title{
Thermal Imaging of Nanostructures by Quantitative Optical Phase Analysis
}

Guillaume Baffou, Pierre Bon, Julien Savatier, Julien Polleux, Min Zhu, Marine Merlin, Herve Rigneault, Serge Monneret

\section{To cite this version:}

Guillaume Baffou, Pierre Bon, Julien Savatier, Julien Polleux, Min Zhu, et al.. Thermal Imaging of Nanostructures by Quantitative Optical Phase Analysis. ACS Nano, 2012, 6 (3), pp.2452 - 2458. 10.1021/nn2047586 . hal-00729073

\section{HAL Id: hal-00729073 https://hal.science/hal-00729073}

Submitted on 7 Sep 2012

HAL is a multi-disciplinary open access archive for the deposit and dissemination of scientific research documents, whether they are published or not. The documents may come from teaching and research institutions in France or abroad, or from public or private research centers.
L'archive ouverte pluridisciplinaire HAL, est destinée au dépôt et à la diffusion de documents scientifiques de niveau recherche, publiés ou non, émanant des établissements d'enseignement et de recherche français ou étrangers, des laboratoires publics ou privés. 


\title{
Thermal Imaging of Nanostructures
}

\section{by Quantitative Optical Phase Analysis}

\author{
Guillaume Baffou ${ }^{1},{ }^{*, \dagger}$ Pierre Bon ${ }^{1,2},{ }^{\dagger}$ Julien Savatier ${ }^{1},{ }^{\dagger}$ Julien Polleux ${ }^{3,4},{ }^{\dagger}$ Min \\ Zhu $^{3},{ }^{\dagger}$ Marine Merlin ${ }^{1},{ }^{\dagger}$ Hervé Rigneault $1{ }^{\dagger}$ and Serge Monneret ${ }^{1 \dagger}$ \\ ${ }^{1}$ Institut Fresnel, UMR CNRS 6133, Domaine Universitaire Saint-Jérôme, 13397 Marseille, \\ France, ${ }^{2}$ PHASICS SA, Campus de l'Ecole Polytechnique, 91128 Palaiseau, France, ${ }^{3}$ Max \\ Planck Institute of Biochemistry, Department of Molecular Medicine, 82152 Martinsried, \\ Germany, and ${ }^{4}$ Max Planck Institute for Intelligent Systems, Department of New Materials and \\ Biosystems, 70569 Stuttgart, Germany \\ E-mail: guillaume.baffou@fresnel.fr
}

\begin{abstract}
We introduce an optical microscopy technique aiming at characterizing the heat generation arising from nanostructures, in a comprehensive and quantitative manner. Namely, it permits to i) map the temperature distribution around the source of heat, ii) map the heat power density delivered by the source and iii) retrieve the absolute absorption cross section of light-absorbing structures. The technique is based on the measure of the thermal-induced refractive index variation of the medium surrounding the source of heat. The measurement is achieved using an association of a regular CCD camera along with a modified Hartmann diffraction grating. Such a simple association makes this technique straightforward to implement on any conventional microscope with its native broadband illumination conditions. We illustrate this technique on gold nanoparticles illuminated at their plasmonic resonance. The spatial resolution of this technique is diffraction limited and temperature variations weaker than $1 \mathrm{~K}$ can be

\footnotetext{
${ }^{*}$ To whom correspondence should be addressed

${ }^{\dagger 1}$ Institut Fresnel, UMR CNRS 6133, Domaine Universitaire Saint-Jérôme, 13397 Marseille, France

$\$ 2$ PHASICS SA, Campus de l’Ecole Polytechnique, 91128 Palaiseau, France

I[3 Max Planck Institute of Biochemistry, Department of Molecular Medicine, 82152 Martinsried, Germany

$\$ 4$ Max Planck Institute for Intelligent Systems, Department of New Materials and Biosystems, 70569 Stuttgart, Germany
} 
detected.

keywords: Microscopy, thermodynamics, phase imaging, plasmonics, Gold nanoparticles

Probing temperature at the nanoscale is fundamentally a complicated task mainly because heat has a non-propagative nature, unlike light. Yet, the ability to investigate thermal-induced phenomena on the nanoscale would help developing unprecedented applications in many active current areas of research, ${ }^{1}$ namely photothermal cancer therapy, ${ }^{2,3}$ drug release, ${ }^{4}$ nano-surgery, ${ }^{5}$ opto-acoustic imaging, ${ }^{6,7}$ nanochemistry, ${ }^{8,9}$ micro-fluidics, ${ }^{10}$ magnetic recording ${ }^{11}$ and thermonics. ${ }^{12}$ Twenty years ago, as a temperature microscopy technique, the use a sharp hybrid tip as a nano-thermocouple was proposed. ${ }^{13,14}$ Even though such an approach achieved a spatial resolution of $50 \mathrm{~nm}$, it appeared to be very invasive due to the proximity of the tip to sample. More recently, several thermal microscopy technique have been developed that are much less invasive since they are based on far-field optical measurements. These optical techniques rely either on fluorescence intensity, ${ }^{15,16}$ fluorescence polarization anisotropy, ${ }^{17,18}$ fluorescence spectroscopy, ${ }^{19-21}$ Raman spectroscopy, ${ }^{22}$ infrared spectroscopy ${ }^{23}$ or X-ray spectroscopy ${ }^{24}$ measurements. However, all these techniques suffer from drawbacks that make them suited only for particular cases. Namely, they suffer from a slow read-out rate, ${ }^{19-22}$ a low resolution, ${ }^{23}$ a lack of reliability, ${ }^{15,16}$ a poor temperature sensitivity ${ }^{22}$ or the necessity to modify (tag) the medium with fluorescent molecules or nanocrystals. $^{15-21}$

In this paper, we propose a thermal microscopy technique sensitive to the thermal-induced variation of the refractive index of the medium of interest. This optical technique circumvents the limitations of all the previous techniques mentioned above and is straightforward to implement on any conventional optical microscope. We explain how it leads to a quantitative real-time retrieval of the distributions of both the temperature and the source of heat with a sub-micrometric resolution. The theory and the retrieval algorithms are detailed. We chose to illustrate the capabilities of this technique on gold nanoparticles (NPs), which act as ideal nanosources of heat when illuminated at their plasmonic resonance frequency. Moreover, we show how a quantitative measurement of the actual absorption cross section can be achieved whatever the nature of the absorbing structure. We chose to name this technique TIQSI for Thermal Imaging using Quadriwave Shearing Interferometry. 


\section{Results and discussion}

We consider in this work a two-dimensional distribution of absorbers (gold nanoparticles) distributed at the interface between a solid (glass substrate) and a liquid environment (like water). When the absorbers are illuminated, heat is generated and a temperature steady state profile appears over a time scale that depends on the spatial extension of the source of heat (typically below $10 \mu$ s for structures smaller than $1 \mu \mathrm{m}$, in water ${ }^{25}$ ). This temperature increase is responsible for a variation of the refractive index of the surrounding liquid over a typical length much larger than the size of the particle itself. This effect has been recently exploited independently by the groups of Lounis and Orrit as a localization technique of NPs or single molecules. ${ }^{26-29}$ NPs and molecules of interest in these works were non-luminescent and far too small to scatter any incident light, making them invisible using any conventional optical technique. To circumvent this limitation, the idea developed in these groups was to exploit the light absorption capabilities of the nano-objects to thermally induce an extended variation of the refractive index of the surroundings, much easier to detect than the nano-objects themselves. However, this localization technique is based on the modulation of the heating laser to achieve an heterodyne detection. For this reason, it cannot be used to measure any steady-state temperature distribution. Moreover, this technique remains slow and requires a complex experimental configuration comprising an acousto-optical modulator and a heterodyne detection.

Recently, our group has developed an optical phase microscopy technique capable of mapping quantitatively the optical path difference (OPD) through a refractive object in real time with a sensitivity of $\sim 1$ nm. ${ }^{30}$ This technique was illustrated by imaging microscopic dielectric beads and living organisms. The experimental approach is based on the use of a quadriwave lateral shearing interferometer as a wave front analyzer (WFA) (see Supplementary Information for more details). Basically, it consists of a modified Hartmann grating (MHG) associated with a regular CCD camera. The device, named Sid4Bio ${ }^{\circledR}$, was purchased from Phasics SA. ${ }^{31}$ The interferogram produced by the MHG and recorded by the CCD camera can be processed in real time to retrieve the OPD. Interestingly, this interferometric technique is achromatic and can be mounted on any conventional white-light transmission microscope. In the present work, we demonstrate how this experimental approach can be the basis of an efficient and easy-to-implement thermal imaging technique. The experimental setup is detailed in Figure 1. All the samples we used consist of a sandwich structure where a liquid layer of thickness $h$ is limited by two glass coverslips in the $z$ direction. The gold nanoparticles acting as nanosources of heat are lying on the bottom coverslip.

When experiencing a temperature variation $\delta T(\mathbf{r})=T(\mathbf{r})-T_{\infty}$, a given medium undergoes a variation 


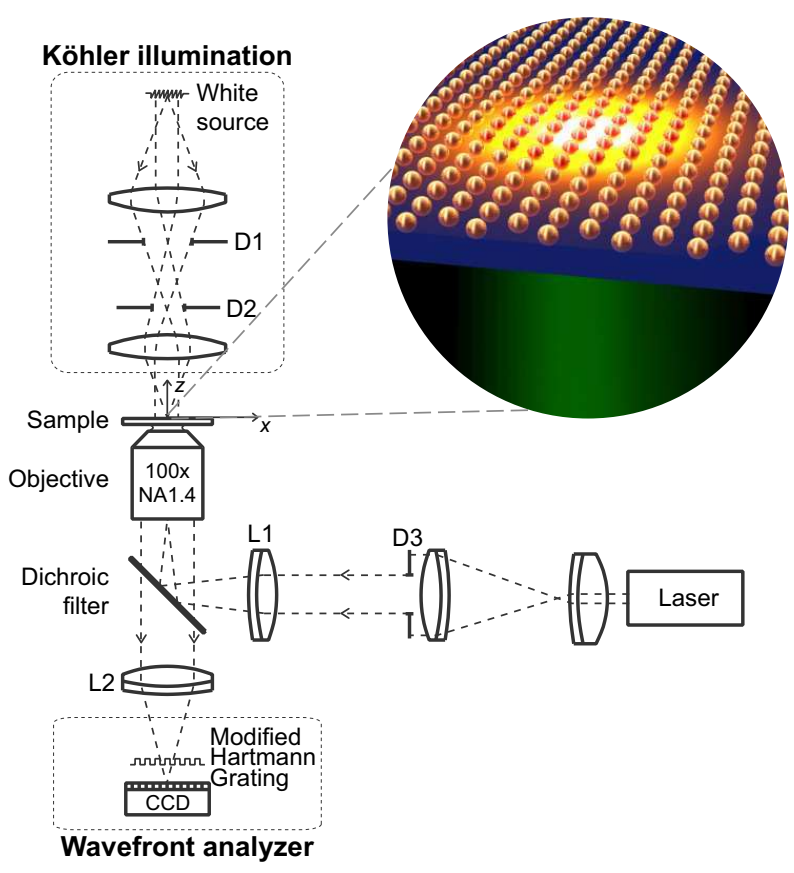

Figure 1: Schematic of the experimental setup. The sample is illuminated using a Köhler configuration with a reduced numerical aperture (D2) to increase the spatial coherence of the light. A laser is used to excite the gold structures at their plasmonic resonance and heat them (either at 532 or $808 \mathrm{~nm}$, depending of the sample). It is focused at the objective entrance pupil by L1 to perform a wide field illumination. A diaphragm D3 is used to control the beam diameter and to achieve a uniform illumination at the sample location. This diaphragm is optically conjugated with the sample by the objective/L1 association The sample is imaged onto a CCD camera by L2 through a modified Hartmann grating. This creates an interferogram on the CCD that is processed to retrieve the optical path difference generated by the thermalinduced refractive index variation.

$\delta n(\mathbf{r})$ of its refractive index. This variation is usually described by a Taylor development of the $N^{\text {th }}$ order: $^{32}$

$$
\delta n=\sum_{j=1}^{N} \beta_{j} \delta T^{j} .
$$

where $\beta_{j}$ are empirical parameters (see Supplementary information or Ref. ${ }^{32}$ for values). For the sake of simplicity, we shall consider in this theoretical part only the first (linear) term of this Taylor series: $\delta n=\beta_{1} \delta T$. In water, this assumption is valid if the temperature increase over the ambient temperature does not exceed $\sim 20^{\circ} \mathrm{C} .{ }^{32}$ It makes the equations linear and simplifies the algorithm used to retrieve the temperature profile. When higher temperature increases are considered, a non-linear iterative numerical procedure is required. The approach is more complex but still simple to implement as detailed in supplementary information.

To begin with, let us consider a point-like source of heat located at the solid-liquid planar interface 
at $(x, y, z)=(0,0,0)$. In the surrounding liquid, the temperature distribution is governed by the Poisson equation:

$$
\kappa \nabla^{2} T(\mathbf{r})=P_{0} \delta(\mathbf{r})
$$

where $\kappa$ is the thermal conductivity of the surrounding liquid $(0.6 \mathrm{~W} / \mathrm{m} / \mathrm{K}$ for water $), T(\mathbf{r})$ the temperature and $P_{0}$ the power absorbed (i.e. delivered) by the point-like source of heat. In spherical coordinates, the solution reads:

$$
\begin{aligned}
T(r) & =T_{\infty}+\frac{P_{0}}{4 \pi \kappa r}=T_{\infty}+\frac{\bar{P}_{0}}{r}, \\
T(r) & =T_{\infty}+\bar{P}_{0} G_{T}(r)
\end{aligned}
$$

where $T_{\infty}$ is the ambient temperature, $\bar{P}_{0}=P_{0} / 4 \pi \kappa$ is the normalized power and $G_{T}(r)=1 / r$ the standard Green's function associated to the Poisson equation.

Experimentally, we use the WFA to measure two phase images of a given area: One reference image acquired (once far all) without heating and one image under heating. The subtraction of these two images provides a measure of the spatial distribution of the optical path difference $\delta \ell(x, y)$ through the liquid layer specifically due to thermal-induced variation of the refractive index distribution $\delta n(x, y, z)$. It can be written as:

$$
\delta \ell(x, y)=\int_{0}^{h} \delta n(\mathbf{r}) \mathrm{d} z=\int_{0}^{h} \beta_{1} \delta T(\mathbf{r}) \mathrm{d} z .
$$

According to Eq. (3), the profile of the temperature variation $\delta T$ reads in radial coordinates $(\rho, z)$ :

$$
\delta T(\rho, z)=\frac{\bar{P}_{0}}{\sqrt{\rho^{2}+z^{2}}}
$$

Using Eqs. (5) and (6) and after integration, the OPD reads:

$$
\begin{aligned}
& \delta \ell(\rho)=\bar{P}_{0} \beta_{1} \ln \left(h / \rho+\sqrt{1+(h / \rho)^{2}}\right), \\
& \delta \ell(\rho)=\bar{P}_{0} \beta_{1} \sinh ^{-1}(h / \rho)=\bar{P}_{0} G_{\ell}(\rho),
\end{aligned}
$$

where $G_{\ell}(\rho)=\beta_{1} \sinh ^{-1}(h / \rho)$ stands for the Green's function for the phase distribution, since the source 
of heat is a Dirac distribution. By eliminating the radial coordinate $\rho$ in Eqs. (6) and (7), one ends up with a bijective relation between the optical phase difference and the temperature increase at $z=0$ :

$$
\delta T(\rho, 0)=\frac{\bar{P}_{0}}{h} \sinh \left(\frac{\delta \ell(\rho)}{\bar{P}_{0} \beta_{1}}\right) .
$$

Note that this formula is only valid for a point-like source of heat, since it was derived using formula (6). In the most general case (extended and non-uniform source of heat), such a bijective relation does not exist between the OPD and the temperature and an inversion procedure is required, which we shall detail now.

Consider now an extended non-uniform source of heat characterized by a delivered heat power density (HPD) $p(x, y)=4 \pi \kappa \bar{p}(x, y)$. The subsequent OPD and temperature distributions can be expressed as convolutions with the associated Green's functions:

$$
\begin{aligned}
\delta \ell(x, y) & =\left[\bar{p} \otimes G_{\ell}\right](x, y), \\
\delta T(x, y) & =\left[\bar{p} \otimes G_{T}\right](x, y),
\end{aligned}
$$

which turns into simple multiplications in the Fourier domain:

$$
\begin{aligned}
\tilde{\delta} \ell(\mathbf{k}) & =\tilde{\bar{p}}(\mathbf{k}) \tilde{G}_{\ell}(\mathbf{k}) \\
\tilde{\delta T}(\mathbf{k}) & =\tilde{\bar{p}}(\mathbf{k}) \tilde{G}_{T}(\mathbf{k}),
\end{aligned}
$$

where $\tilde{f}$ stands for the Fourier transform of $f$. From the experimental phase image $\delta \ell(x, y)$, the heat source distribution $\bar{p}(x, y)$ can be simply retrieved using Eq. (12): $\tilde{\bar{p}}(\mathbf{k})=\tilde{\boldsymbol{\delta} \ell}(\mathbf{k}) / \tilde{G}_{\ell}(\mathbf{k})$ followed by an inverse Fourier transform. However, such a basic approach is likely to cause divergence problems in case of low signal to noise ratio in the OPD images. This standard deconvolution issue is well-known and different approaches have been proposed in the literature. Among them, one of the simplest is the Tikhonov regularization method, ${ }^{33}$ which we used here. Its principle is detailed in supplementary information. Then, the temperature distribution $T(x, y)$ is retrieved using Eqs. (13) followed by an inverse Fourier transform.

The spatial resolution of the TIQSI technique is given by $\lambda / N A$ (since the illumination is spatially coherent) where $\lambda$ is the characteristic wavelength of the Köhler illumination and NA the numerical aperture of the objective. Our set of measurements corresponds to $\lambda=650 \mathrm{~nm}$ and NA $=1.4$, which leads to a theoretical spatial resolution of around $450 \mathrm{~nm}$. The frame rate of our CCD camera is $10 \mathrm{~Hz}(100 \mathrm{~ms}$ per 


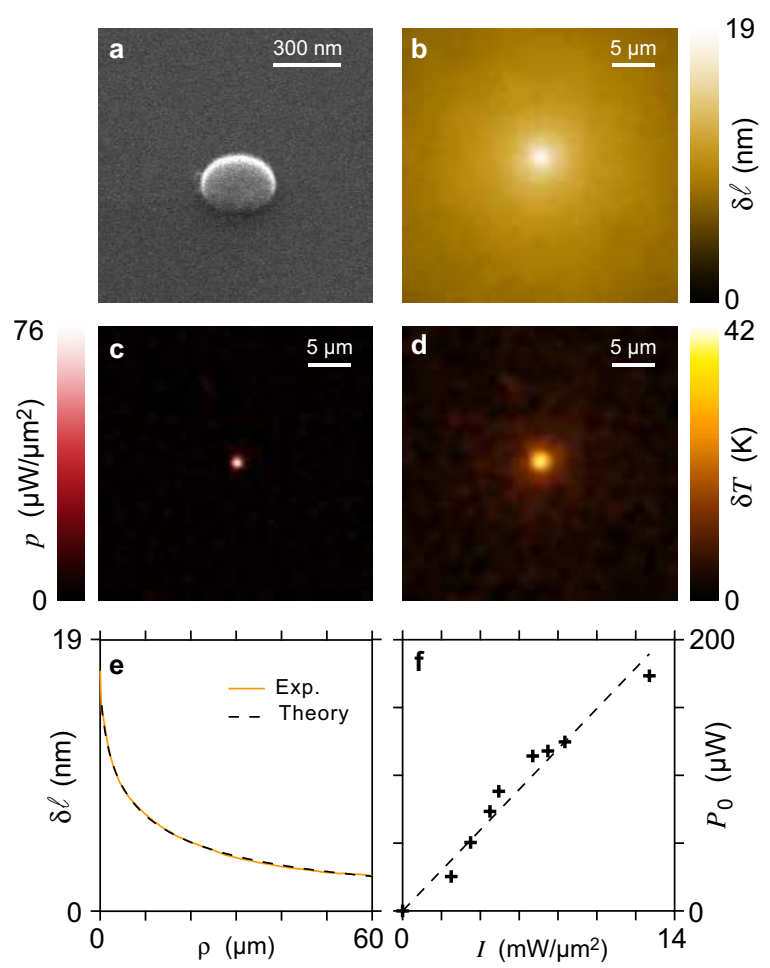

Figure 2: a) Scanning Electronic Microscope (SEM) image of the gold structure under study. This structure was illuminated at a wavelength $\lambda_{0}=808 \mathrm{~nm}$. b) Raw image of $\delta \ell(x, y), I=12.8 \mathrm{~mW} / \mu \mathrm{m}^{2}$. c) Heat power density $p(x, y)$ processed from image $\mathrm{b}$ using the NL algorithm. d) Temperature distribution $\delta T(x, y)$ processed from image c using Eq. (11). e) Measured radial profile of the OPD distribution in image $b$ (solid line), fitted using Eq. (9) (dashed line). f) Delivered power $P_{0}$, as function of the laser irradiance, measured by fitting the OPD radial profile using Eq. (9) with $P_{0}$ and $h$ as the unknown parameters.

image). We usually average a series of 10 to 30 frames to improve the signal to noise ratio. Hence, one usually needs a few seconds per image. This is much faster than most of the other thermal microscopy techniques that need a few minutes or even a few hours to record an image. This readout rate is not a fundamental limitation of the TIQSI technique and it could be improved with a faster and better cooled CCD camera.

We shall first present and discuss experimental results obtained on a point-like source of heat, i.e. a gold disc, $300 \mathrm{~nm}$ in diameter and $40 \mathrm{~nm}$ thick illuminated by a laser beam at $\lambda_{0}=808 \mathrm{~nm}$ (Figure 2a). Figure $2 \mathrm{~b}$ displays the raw image of the OPD measured by the WFA. From this image, we have applied the Tikhonov algorithm to retrieve the actual HPD and temperature distributions, as displayed in Figure 2cd. Interestingly, the temperature extension (Figure $2 \mathrm{~d}$ ) is much narrower that the extension of the OPD distribution. This observation is consistent with the $-\ln (\rho)$ decrease of the OPD Green's function (Eq. (7)), compared to the much steeper $1 / \rho$ decrease of the temperature Green's function (Eq. (4)). In this 
measurement, 30 frames of $100 \mathrm{~ms}$ were averaged. The standard deviation of the noise in the raw OPD image (Figure 2b) is $0.15 \mathrm{~nm}$ and in the temperature image (Figure 2d), $1.1 \mathrm{~K}$.

In experimental optics, determining the actual absorption cross section of a unique absorber is not straightforward mainly because the absorption process is precisely not associated with any light reemission that could contain information and be detected in the far-field (unlike extinction or scattering cross-sections measurements). Optical techniques to measure absorption cross sections have been proposed recently. ${ }^{34,35}$ However, all of them suffer from at least one of these drawbacks: i) They consist in fact in measuring extinction instead of absorption. This assimilation is valid only for small particles, i.e. when scattering is negligible (extinction=absorption+scattering), ii) They do perform absorption spectroscopy but they do not yield a quantitative measurement of the cross section. In this second case, a quantitative ACS can be eventually estimated indirectly by comparing the photothermal signal with the one obtained on a reference absorber with a supposedly known ACS. ${ }^{29}$ Our approach circumvent these two limitations at the same time: It allows to get a direct quantitative measurement of the actual absorption cross section $\sigma_{\text {abs }}$ without any assumption and independently of the nature of the absorber. One just needs to estimate the absorbed power $P_{0}$ and then calculate $\sigma_{\mathrm{abs}}=P_{0} / I$ where $I$ is the irradiance of the laser beam (power per unit area). There are two possibilities to estimate $P_{0}$. The first one consists in fitting the radial profile of the OPD image (as shown in Figure 2e) using formula (7) with $P_{0}$ (and eventually $h$ ) as the unknown parameter. This first approach gives in the case of Figure 2: $\sigma_{\mathrm{abs}}=14930 \pm 530 \mathrm{~nm}^{2}$. The second approach consists in estimating $P_{0}$ by a spatial integration of the HPD image (Figure 2c). It naturally yields a very similar result: $\sigma_{\mathrm{abs}}=15300 \pm 600 \mathrm{~nm}^{2}$. Calculations using the Green's dyadic tensor method ${ }^{18}$ on a gold disc, $288 \mathrm{~nm}$ in diameter, $39 \mathrm{~nm}$ thick, discretized using 2066 cells and illuminated at lambda=808 nm, led to $\sigma_{\text {abs }}=17200 \mathrm{~nm}^{2}$, which is the good order of magnitude. The difference of $14 \%$ with the experimental measurement can be mainly explained by the fact that the real structure is not a perfect disc. The accuracy of the measurement can be optimized by acquiring a set of images at different irradiances, as illustrated in Figure 2f. The first approach is handy since it does not require the computation of the HPD distribution, while the second approach is more general and applies even for extended (not point-like) sources of heat. Note that one does not need to optically resolve the NP to measure its ACS. Indeed, the ACS retrieval procedure is based on the fit of the logarithmic OPD profile around the particle that extends to infinity, even for nanometric/point-like particles (see Figure 2a-c). 


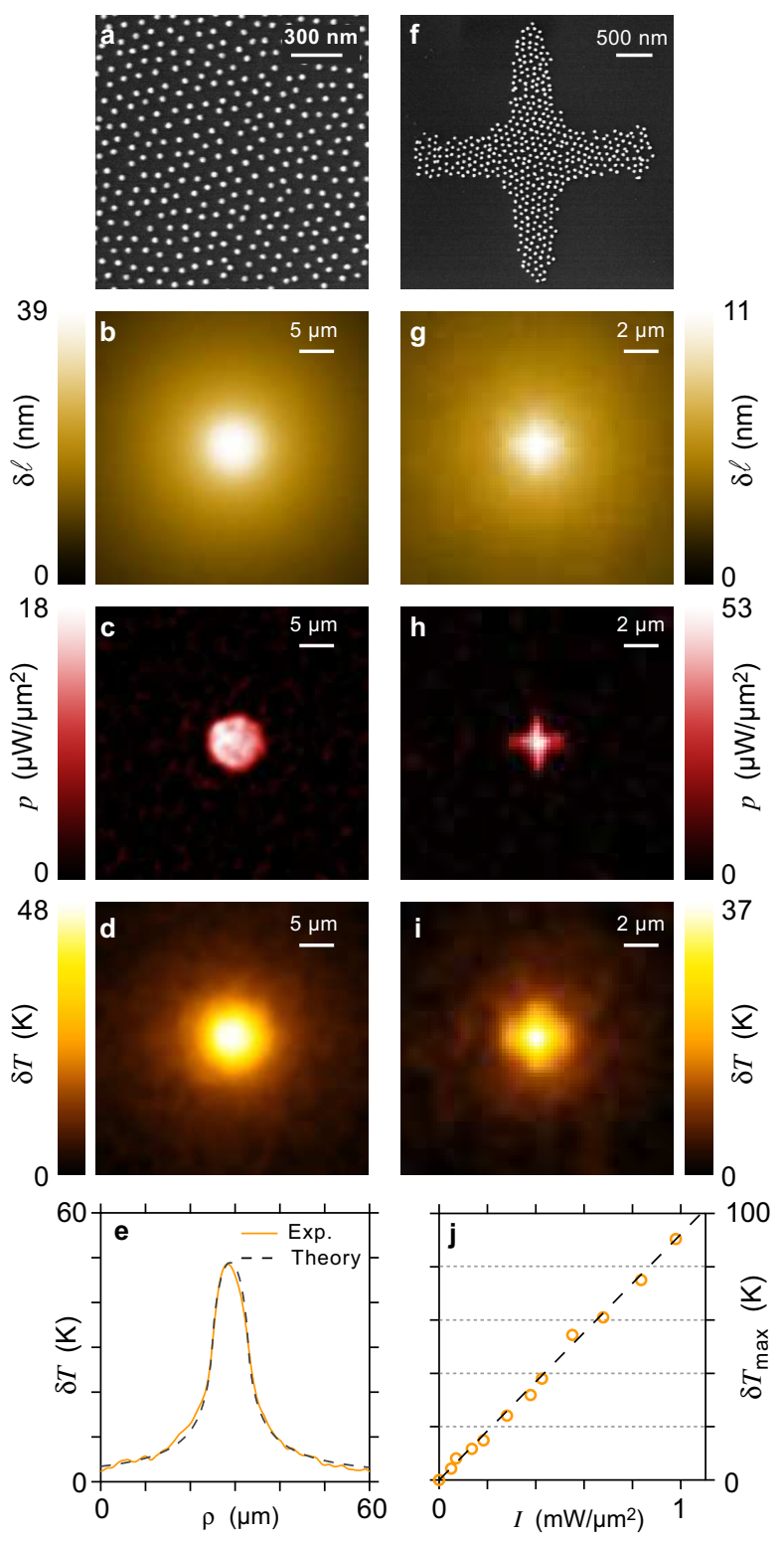

Figure 3: a) SEM image displaying a quasi-hexagonally ordered array of gold nanoparticles. b) Raw image of the measured optical path difference. Heating is performed by a circular beam of diameter $D=8.0 \mu \mathrm{m}$ and uniform irradiance $105 \mu \mathrm{W} / \mu \mathrm{m}^{2}$ at $\lambda=532 \mathrm{~nm}$. c) Processed heat power density. d) Processed temperature increase. e) Radial profile of the temperature distribution. f) Optical image of the micropatterned array of gold nanodots. A SEM image in the inset displays their organization. This whole structure is illuminated by a uniform beam of irradiance $250 \mu \mathrm{W} / \mu \mathrm{m}^{2}$. g) Raw image of the measured optical path difference. h) Heat source density. i) Temperature increase. j) Maximum temperature of the microstructure as function of the laser irradiance.

We shall now present experimental results that address the situation of an extended distribution of heat sources. In Figure 3, experiments have been carried out on an array of quasi-hexagonally organized NPs. The sample has been done by block-copolymer lithography. ${ }^{36}$ It consists of spherical gold NPs, $d=30 \pm 3$ $\mathrm{nm}$ in diameter and separated by $p=100 \pm 10 \mathrm{~nm}$. The NP interdistance is small enough compared to the resolution of the microscope to consider the HPD as a continuous physical quantity. The heat is gen- 
erated by a laser beam at $\lambda_{0}=532 \mathrm{~nm}$, close to the resonance wavelength of the gold particles. In the first case, the NP array covers the whole sample (Figure 3a) and the laser illuminates uniformly a circular area of diameter $8.0 \mu \mathrm{m}$. In the second case, NP distributions form cross-like structures (Figure 3f). One of them is uniformly illuminated by the heating laser. From the optical path difference measured by the WFA (Figure 3b-g) the distribution of the HPD is computed using the Tikhonov inversion algorithm. As expected, the heat source distributions (Figure 3c-h) are well delimited while the temperature distributions tend to spread out (Figure 3d-i). In order to verify the consistency of the temperature distribution, we performed numerical simulation of the temperature profile in Figure 3d using the Green's function formalism ${ }^{25}$ (Figure 3e). The input parameters regarding the geometry and the illumination conditions were $I=105 \mu \mathrm{W} / \mu \mathrm{m}^{2}, D=8.0 \mu \mathrm{m}, p=105 \mathrm{~nm}, d=28 \mathrm{~nm}$. The only variable parameters were $d$ and $p$ and remain very close to the measured values on the SEM image. The matching is very good as observed in Figure 3e, which reinforces the validity of our post-processing numerical model. A last result that we wish to share is presented in Figure 3j. It shows that, as expected, the temperature increase is proportional to the laser irradiance, even over a wide range of temperature. The non-linearities of the problem are thus well taken into account in our numerical procedure (see Supplementary information).

In this last paragraph, we wish to discuss the temperature sensitivity that the TIQSI method can achieve. Figure 4 presents a measurement performed on an extended quasi-hexagonal array of nanodots. The structure is illuminated by a uniform circular beam of diameter $11 \mu \mathrm{m}$ and and power $P=0.35$ $\mathrm{mW}$. Such a low irradiance $\left(I=3.7 \mu \mathrm{W} / \mu \mathrm{m}^{2}\right)$ led to a fairly noisy OPD image (Figure $4 \mathrm{a}$ ) due to a weak temperature increase. Processing of the OPD image led to a temperature image presented in Figure 4b and revealed that the temperature increase was about $1 \mathrm{~K}$. One can see that such a temperature increase is perfectly visible on the OPD image, even though it leads to a very noisy temperature image. The standard deviation of the noise in the OPD image (Figure 4a) is $0.044 \mathrm{~nm}$ and in the temperature image (Figure 4b), $0.36 \mathrm{~K}$. The higher noise level in the temperature image compared with the OPD image is inherent to the deconvolution procedure, and mostly features low frequencies. Below a temperature increase of $1 \mathrm{~K}$, it is hard to obtain a well-defined temperature map. However, since most of the noise observed on the interferogram recorded by the CCD image is due to dark current, it could be possible to increase the signal to noise ratio and reach even better temperature sensitivity by increasing the number of accumulated frames, or by using a better cooled CCD camera. Note that no physical limitation exists on the temperature accuracy, unlike the spatial resolution that is bound to remain diffraction-limited. 

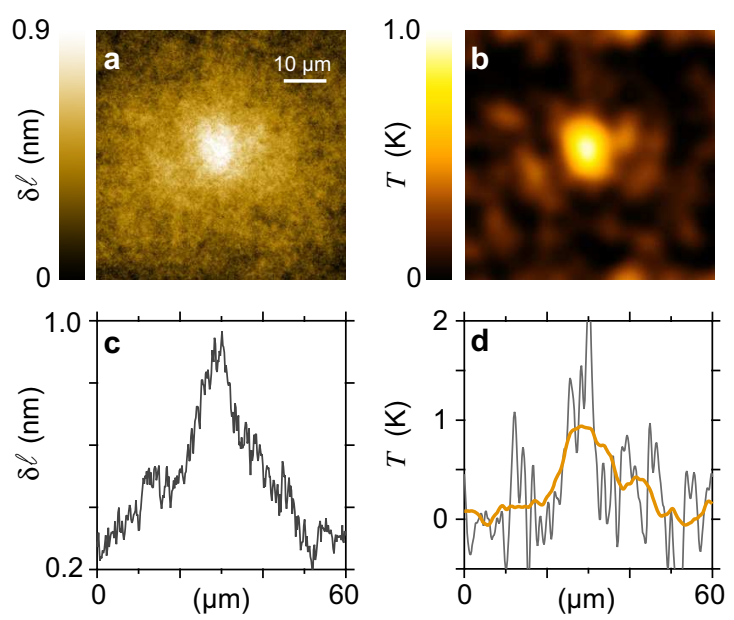

Figure 4: a) Raw thermal-induced OPD image obtained on a quasi-hexagonally ordered array of gold nanoparticles (see Figure 3a). b) Processed temperature distribution (smoothed using a 10-pixel Gaussian function). c) Cross section of image (a). Cross section of the raw image (b) without smoothing (dash line) and with smoothing (solid line).

\section{Conclusion}

In summary, we introduced a thermal microscopy technique sensitive to thermal-induced refractive index variation. The measurement is achieved using a simple experimental configuration consisting of a Hartmann diffraction grating attached to a CCD camera. This technique cumulates many valuable advantages compared with previous thermal imaging techniques: i) It is fast. According to the CCD frame rate, it takes around one second to acquire an acceptable image in most cases. ii) No scanning is required. It is a one-shot measurement of a 2D image. iii) It has a diffraction limited resolution (less than 500 $\mathrm{nm})$. iv) No modification of the sample is required, like using fluorescent markers. It works whatever the sample as far as there is a liquid environment. v) No need to use sophisticated devices such as heterodyne detection, acousto-optic modulator, spectrometer, etc, like previous thermal imaging techniques. All the thermal measurements can be performed by plugging in the $\mathrm{CCD}$ /grating device on a conventional optical microscope, just like a regular CCD camera. vi) The TIQSI techniques features an un precedented versatility since it can quantitatively measure temperature, heat source density and absorption cross sections of nanoparticles and nanostructures. We chose to illustrate the abilities of the technique on gold NPs, which stand for ideal nano-sources of heat. However, we wish to emphasize that the method is not restricted to metal NPs and can be extended to any 2D-distribution of heat sources, independently on their nature. Moreover, this technique is straightforward to implement on any conventional microscope. For these reasons, pending important applications are envisioned not only in plasmonics but also in areas such 
as nano-electronics, micro-fluidics or cell biology.

\section{Methods}

Fabrication of the quasi-hexagonal arrays of gold nanodots: In a typical synthesis, polystyrene(1056)-block-poly(2-vinylpyridine)(495) (PS1056-b-P2VP495) from Polymer Source Inc. was dissolved at room temperature in anhydrous toluene (Sigma-Aldrich) with a concentration of $5 \mathrm{mg} / \mathrm{mL}$ and stirred for 2 days. The quantity of gold precursor was calculated relative to the number of $\mathrm{P} 2 \mathrm{VP}$ units with a loading parameter equal to 0.5 , i.e., 1 molecule of $\mathrm{HAuCl}_{4}$ for $2 \mathrm{P} 2 \mathrm{VP}$ monomers. Hydrogen tetrachloroaurate (III) trihydrate $\left(\mathrm{HAuCl}_{4} \cdot 3 \mathrm{H}_{2} \mathrm{O}\right.$, Sigma-Aldrich) was added to the $\mathrm{dBCP}$ solution and stirred for 2 days in a sealed glass vessel. Glass cover slips (Carl Roth) were cleaned in a piranha solution for at least 5 hours and were extensively rinsed with MilliQ water and dried under a stream of nitrogen. Micellar monolayers were prepared by dip-coating a glass cover slip into the previously prepared solutions with a constant velocity equal to $24 \mathrm{~mm} / \mathrm{min}$. To remove the organic template and to form inorganic nanoparticles, the dip-coated glass slides were exposed to oxygen plasma ( $150 \mathrm{~W}, 0.15 \mathrm{mbar}, 45 \mathrm{~min}$, PVA TEPLA 100 Plasma System). In the case of the micropatterned arrays of gold nanodots, a 1- $\mu \mathrm{m}$ thick layer of AR-P5350 photoresist (Allresist $\mathrm{GmbH}$ ) was spin-coated onto the arrays. Illumination was performed under a maskaligner (Suss Microtec $\mathrm{GmbH}$ ) with a dose of $175 \mathrm{~mJ} / \mathrm{cm}^{2}$ from a HBO 350 mercury lamp and the microstructures were developed according to the manufacturer's instructions. ${ }^{36}$ The enlargement of gold nanodots was performed in an aqueous solution containing hydroxylamine $(0.02$ $\mathrm{mM})$ and $\mathrm{HAuCl}_{4}(0.1 \mathrm{wt} \%)$ for $10 \mathrm{~min}$. Scanning electron measurements were performed with a Dual BeamTM (FIB/SEM) instrument (Quanta 3D FEG, FEI, Hillsboro).

Acknowledgment: We acknowledge fruitful discussions with Philippe Réfrégier. We thank the Institute of Photonic Sciences (ICFO) for providing the lithographic sample (Figure 2a). We acknowledge financial support from the region ProvenceAlpes-Cote d'Azur, the Fonds Unique Interministériel (QuITO project), the Centre National de la Recherche Scientifique, the $7^{\text {th }}$ Framework Program of the European Community (FP7) through the Marie Curie Action for career development (IEF "Multi-PGNAs") and the Max Planck Society.

\section{TOC image:}
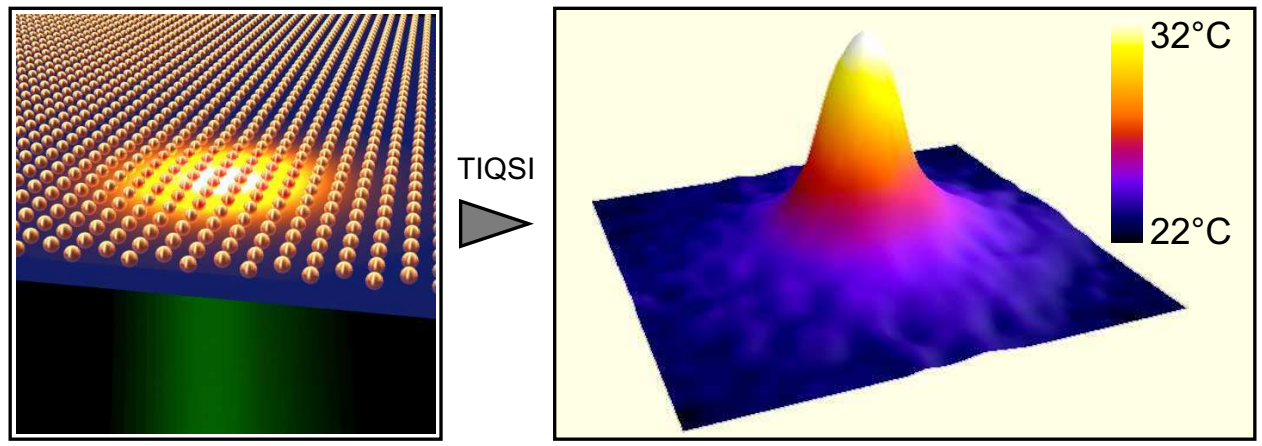


\section{References}

1. Govorov, A. O.; Richardson, H. H. Generating Heat with Metal Nanoparticles. Nano Today 2007, 2, 30.

2. Lal, S.; Clare, S. E.; Halas, N. J. Nanoshell-Enabled Photothermal Cancer Therapy: Impending Clinical Impact. Acc. Chem. Res. 2008, 41, 1842.

3. Jain, P. K.; El-Sayed, I. H.; El-Sayed, M. A. Au Nanoparticles Target Cancer. Nano Today 2007, 2, 18.

4. Han, G.; Ghosh, P.; De, M.; Rotello, V. M. Drug and Gene Delivery using Gold Nanoparticles. NanoBioTechnology 2007, 3,40 .

5. Urban, A.; Pfeiffer, T.; Fedoruk, M.; Lutich, A.; Feldmann, J. Single Step Injection of Gold Nanoparticles through Phospholipid Membranes. ACS Nano 2011, 5, 3585-3590.

6. Eghtedari, M.; Oraevsky, A.; Copland, J.; Kotov, N.; Conjusteau, A.; Motamedi, M. High Intensity of in vivo Detection of Gold Nanorods Using a Laser Optoacoustic Imaging System. Nano Lett. 2007, 7, 1914-1918.

7. Beard, P. Biomedical Photoacoustic Imaging. Interface Focus 2011, 1, 602.

8. Jin, C.; Li, Z.; Williams, R.; Lee, K.; Park, I. Localized Temperature and Chemical Reaction Control in Nanoscale Space by Nanowire Array. Nano Lett. 2011, 11, 4818-4825.

9. Nitzan, A.; Brus, L. E. Theoretical Model for Enhanced Photochemistry on Rough Surfaces. J. Chem. Phys. 1981, 75, 2205 .

10. Donner, J.; Baffou, G.; McCloskey, D.; Quidant, R. Plasmon-Assisted Optofluidics. ACS Nano 2011, 5, year.

11. Challener, W. A.; Peng, C.; Itagi, A. V.; Karns, D.; Peng, W.; Peng, Y.; Yang, X. M.; Zhu, X.; Gokemeijer, N. J.; Hsia, Y.-T. et al. Heat-Assisted Magnetic Recording by a Near-Field Transducer with Efficient Optical Energy Transfer. Nat. Photon. 2009, 3,220.

12. Wang, L.; Li, B. Thermal Memory: A Storage of Phononic Information. Phys. Rev. Lett. 2008, 101, 267203.

13. Pollock, H. M.; Hammiche, A. Micro-Thermal Analysis: Techniques and Applications. J. Phys. D-Appl. Phys. 2001, 34, R23.

14. Sadat, S.; Tan, A.; Chua, Y. J.; Reddy, P. Nanoscale Thermometry using Point Contact Thermocouples. Nano Lett. 2010, $10,2613-2617$.

15. Löw, P.; Kim, B.; Takama, N.; Bergaud, C. High-Spatial-Resolution Surface-Temperature Mapping Using Fluorescent Thermometry. Small 2008, 4, 908.

16. Ross, D.; Gaitan, M.; Locascio, L. E. Temperature Measurement in Microfluidic Systems Using a Temperature-Dependent Fluorescent Dye. Anal. Chem. 2001, 73, 4117. 
17. Baffou, G.; Kreuzer, M. P.; Kulzer, F.; Quidant, R. Temperature Mapping Near Plasmonic Nanostructures Using Fluorescence Polarization Anisotropy. Opt. Express 2009, 17, 3291.

18. Baffou, G.; Girard, C.; Quidant, R. Mapping Heat Origin in Plasmonics Structures. Phys. Rev. Lett. $2010,104,136805$.

19. Vetrone, F.; Naccache, R.; Zamarrón, A.; Juarranz de la Fuente, A.; Sanz-Rodríguez, F.; Martinez Maestro, L.; Martín Rodriguez, E.; Jaque, D.; García Solé, J.; Capobianco, J. A. Temperature Sensing Using Fluorescent Nanothermometers. ACS Nano 2010, 4, 3254.

20. Li, S.; Zhang, K.; Yang, J. M.; Liwei, L.; Yang, H. Single Quantum Dots as Local Temperature Markers. Nano Lett. 2007, 7, 3102-3105.

21. Aiguouy, L.; Tessier, G.; Mortier, M.; Charlot, B. Scanning Thermal Imaging of Microelectronic Circuits with a Fluorescent Nanoprobe. Appl. Phys. Lett. 2005, 87, 184105.

22. Dang, N. C.; Bolme, C. A.; Moore, D. S.; McGrance, S. D. Femtosecond stimulated Raman Scattering Picosecond Molecular Thermometry in Condensed Phases. Phys. Rev. Lett. 2001, 107, 043001.

23. Sade, S.; Nagli, L.; Katzir, A. Scanning Near Field Infrared Radiometry for Thermal Imaging of Infrared Emitters with Subwavelength Resolution. Appl. Phys. Lett. 2005, 87, 101109.

24. Van de Broek, B.; Grandjean, D.; Trekker, J.; Ye, J.; Verstreken, K.; Maes, G.; Borghs, G.; Nikitenko, S.; Lagae, L.; Bartic, C. et al. Temperature Determination of Resonantly Excited Plasmonic Branched Gold Nanoparticles by X-ray Absorption Spectroscopy.

25. Baffou, G.; Rigneault, H. Femtosecond-Pulsed Optical Heating of Gold Nanoparticles. Phys. Rev. B 2011, 84, 035415.

26. Boyer, D.; Tamarat, P.; Maali, A.; Lounis, B.; Orrit, M. Photothermal Imaging of Nanometer-Sized Metal Particles among Scatterers. Science 2002, 297, 1160.

27. Berciaud, S.; Cognet, L.; Blab, G. A.; Lounis, B. Photothermal Heterodyne Imaging of Individual Nonfluorescent Nanoclusters and Nanocrystals. Phys. Rev. Lett. 2004, 93, 257402.

28. Cognet, L.; Berciaud, S.; Lasne, D.; Lounis, B. Photothermal Methods for Single Nonluminescent Nano-Objects. Anal. Chem. 2008, 80, 2288-2294.

29. Gaiduk, A.; Yorulmaz, M.; Ruijgrok, P. V.; Orrit, M. Room-Temperature Detection of a Single Molecule's Absorption by Photothermal Contrast. Science 2010, 330, 353-356.

30. Bon, P.; Maucort, G.; Wattellier, B.; Monneret, S. Quadriwave Lateral Shearing Interferometry for Quantitative Phase Microscopy of Living Cells. Opt. Express 2009, 17, 13080.

31. info@phasics.fr. 
32. Thormählen, I.; Straub, J.; Grigull, U. Refractive Index of Water and its Dependence on Wavelength, Temperature and Density. J. Phys. Chem. Ref. Data 1985, 14, 933-945.

33. Tikhonov, A. N. Solution of Ill-posed problems; Vh Winston, 1977.

34. Arbouet, A.; Christofilos, D.; Del Fatti, N.; Vallée, F.; Huntzinger, J. R.; Arnaud, L.; Billaud, P.; Broyer, M. Direct Measurement of the Single-Metal-Cluster Optical Absorption. Phys. Rev. Lett. 2004, 93, 127401.

35. Celebrano, M.; Kukura, P.; Renn, A.; Sandoghdar, V. Single-Molecule Imaging by Optical Absorption. Nature Photon. 2011, 5, 95-98.

36. Aydin, D.; Schwieder, M.; Louban, I.; Knoppe, S.; Haas, T. L.; Walczak, H.; Spatz, J. P. Micro-Nanostructured Protein Arrays: A Tool for Geometrically Controlled Ligand Presentation. Small 2009, 5, 1014-1018. 